\title{
Anthracene and Pyrene Photooxidation Kinetics in Saltwater Environments
}

Jarod N. Grossman ${ }^{1, \wedge}$, Shawn F. Kowal ${ }^{1, \#}$, Annastacia D. Stubbs ${ }^{1,2}$, Cara N. Cawley ${ }^{1}$, and Tara F. $\operatorname{Kahan}^{1,2 *}$

1. Department of Chemistry, Syracuse University, 1-014 Center for Science and Technology, Syracuse, NY 13244,

2. Department of Chemistry, University of Saskatchewan, 110 Science Place, Saskatoon, SK S7N 5C9, Canada

${ }^{\wedge}$ Currently at Agilent Technologies, CA, USA

\# Currently at Ecology and Environment, NY, USA

*Author to whom correspondence should be addressed: tara.kahan@usask.ca, (306) 966-1168. 110 Science Place, University of Saskatchewan, Saskatoon, Saskatchewan, Canada S7N 5C9

\section{SUPPORTING INFORMATION}

2 Pages

Figures: S1-S2 
Figure S1 shows anthracene and pyrene absorption spectra at wavelengths transmitted by the 295 $\mathrm{nm}$ long-pass cutoff filter that is placed in front of the xenon arc lamp. Spectra were acquired in methanol, as low PAH solubility in water prevents the acquisition of reliable absorbance spectra in the actinic region.

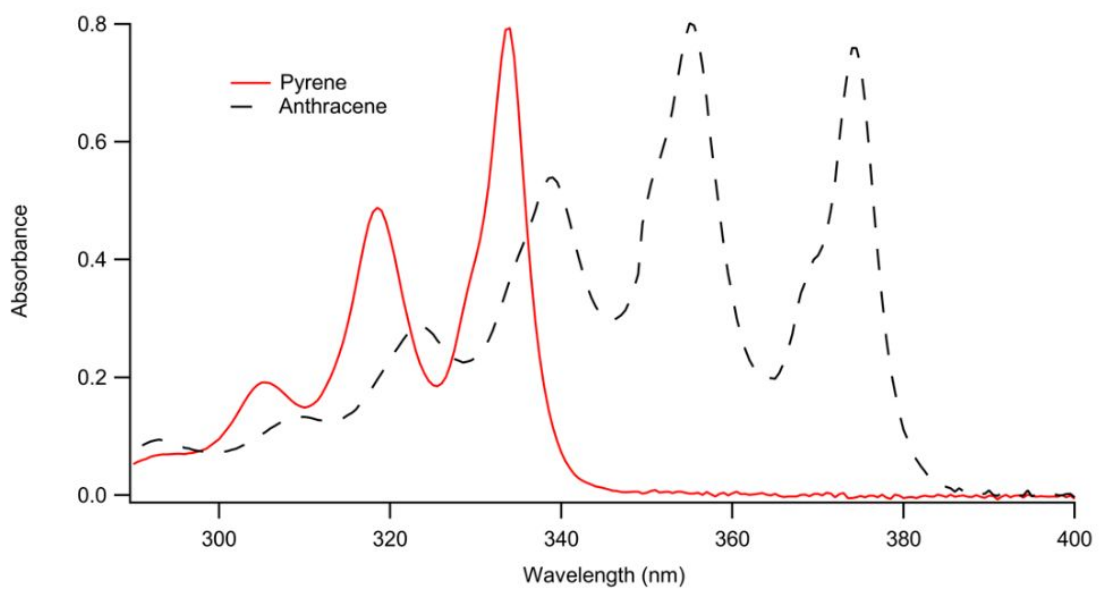

Figure S1. Absorbance spectra of $1.1 \times 10^{-4} \mathrm{M}$ anthracene and $2.0 \times 10^{-5} \mathrm{M}$ pyrene in methanol.

In the manuscript we state that absorbance (and possible subsequent photochemistry) of species in Sargasso Seawater is likely not responsible for observed differences in anthracene rate constants in deionized (DI) water and in Sargasso Sea water. Figure S2 shows that Sargasso Sea water absorbance is negligible at wavelengths in the actinic region. Measured absorbance is well below the spectrometer's limit of detection (which ranges from $\sim 0.001$ at long wavelengths to a maximum of 0.007 at $290 \mathrm{~nm}$ ).

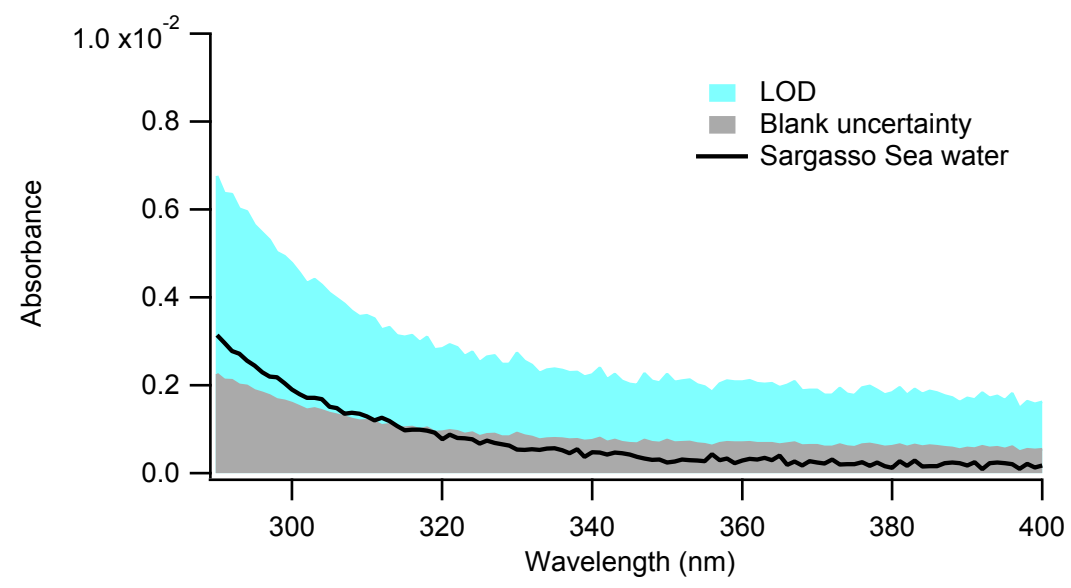

Figure S2. Absorption spectrum of Sargasso seawater. The grey shaded region represents the uncertainty of the blank measurement. The magnitude of the uncertainty is given by the standard deviation about the mean of the absorbance of 3 deionized water samples. The blue shaded region represents the instrument's wavelength-dependent limit of detection (LOD), which is calculated as $3 \times$ the standard deviation. 